\title{
Transpupillary thermotherapy of choroidal melanoma with or without brachytherapy: a dilemma
}

Transpupillary thermotherapy (TTT) is a new approach to the treatment of choroidal melanoma with heat. The early treatment results seem favourable with regard to local tumour control and retained visual acuity; no early metastasis was found..$^{1-3}$ The reported rate of complications is low. ${ }^{23}$

The heat treatment is performed with a temperature calculated at $45-65^{\circ} \mathrm{C}$, induced by an infrared diode laser $(810 \mathrm{~nm})$ with a large beam diameter $(3 \mathrm{~mm})$ and a long exposure time of 1 minute per application. These factors promote a deep penetration of heat into the choroidal melanoma. The result is tumour necrosis up to $3.9 \mathrm{~mm}$ deep and occlusion of blood vessels in the treated area as shown on histopathology. ${ }^{4}$ Depending on the pigmentation of the tumour, the energy is raised or lowered stepwise until the desired effect occurs. The tumour is treated by overlapping applications extending beyond the margin of the tumour. The exposure time should not be shortened to less than 1 minute, as it takes 40 seconds to reach a temperature at subcoagulation level.

TTT is performed as an outpatient procedure and can be easily repeated. Treatment results can be evaluated in several ways. The outcome is favourable when the choroidal melanoma develops into a scar on ophthalmoscopic examination, substantial regression of the tumour thickness is seen on ultrasonography together with an increased reflectivity on the diagnostic A-scan, and hypofluorescence is visible in the treated area on the early and late phases of the fluorescein angiogram. TTT is not indicated in melanoma patients with severe media opacities, in patients with insufficient dilatation of the pupil, or in elevated, peripherally located melanomas. ${ }^{1}$

Simultaneous use of TTT with brachytherapy in the management of melanomas is called "sandwich therapy", where the effect of TTT is maximal at the top of the tumour and brachytherapy efficiently treats the base of the tumour. ${ }^{124}$ Combining both treatments has several advantages. It enabled us to treat patients with tumours thicker than $5 \mathrm{~mm}$, generally the maximum height for ruthenium106 brachytherapy. ${ }^{12}$ Insufficient melanoma regression and/or recurrences after a combined treatment might be retreated with TTT alone. The combination might also lead to a decreased radiation dose of the isotope used in brachytherapy.

In principle, TTT can also be applied as the sole therapy. A satisfactory local tumour control is reported in the early years after TTT as sole therapy for small choroidal melanomas. ${ }^{3}$ TTT as sole therapy seems especially attractive in treating small choroidal melanomas in and around the posterior pole. Visual outcome may be better after TTT than after brachytherapy because the laser beam, but not the radiation beam delivered by the radioactive plaque, can be focused. ${ }^{3}$ As the macular capillaries are very radiosensitive to brachytherapy, this may finally result in radiation maculopathy with a considerable loss of central vision. However, after extramacular applications of sole TTT, macular complications such as transient cystoid macular oedema may be also seen. ${ }^{3}$ A dense scotoma is generally produced by
TTT at the time of application, whereas a scotoma after brachytherapy usually does not develop for up to a year or several years. If heat treatment is performed as sole therapy, effective treatment of the sclera over the tumour is as important as treatment of the tumour itself because intra- and episcleral tumour invasion is present in, respectively, $55.7 \%$ and $8.2 \%$ of patients with medium and large tumours. ${ }^{5}$ In a group of 21 patients with enucleated small melanomas, 17 showed scleral ingrowth on histopathological examination. ${ }^{6}$ Intrascleral melanoma cells surviving treatment might be a focus for tumour recurrence, which is associated with a two to threefold higher risk of metastasis. This is evident from the results after photocoagulation treatment of choroidal melanomas showing a high rate of tumour regrowth. This might be attributed to the low depth of photocoagulation penetration, insufficient to destroy the intrascleral melanoma cells. $^{7}$

In TTT as sole therapy, the question arises as to whether the effect of the heat induced by TTT to the choroidal melanoma is identical to the effect of the heat to the possible melanoma cells in the sclera. In one eye, intentionally enucleated after experimental TTT, total tumour necrosis up to the sclera was visible on histopathological examination. Despite heat damage to the inner layers of the sclera which was evident from scleral oedema and necrosis of sclerocytes, a cluster of tumour cells with a viable aspect was located near the inner border of the sclera. ${ }^{4}$ A recent clinicopathological report on two cases of recurrent juxtapapillary choroidal melanoma after TTT revealed tumour cells appearing histopathologically intact and presumably viable. ${ }^{8}$ One patient was insufficiently treated with TTT because of cataract, another patient was treated with TTT and brachytherapy. Interpreting these data, we have to keep in mind that a pathologist is often unable to guess viability of melanoma cells in an enucleated eye after any treatment, especially in an undertreated eye.

Unfortunately, no sensitive clinical techniques evaluate the effect of TTT on intrascleral and episcleral tumour cells, their destruction, and the early development of extrascleral recurrences. To reduce the risk of insufficient treatment of the sclera and because episcleral tumour recurrences rarely develop as a late complication of brachytherapy, one may consider combining TTT with brachytherapy (the sandwich therapy).

TTT remains an investigative procedure until long term results are available. In the meantime we have to choose between TTT as sole or combined treatment for patients with choroidal melanoma.

J E E KEUNEN

J G JOURNÉE-DE KORVER J A OOSTERHUIS

Department of Ophthalmology, Leiden University Medical Center, Leiden, Netherlands

Correspondence to: Jan EE Keunen, Department of Ophthalmology, Leiden University Medical Center, PO Box 9600, 2300 RC Leiden, Netherlands 
1 Oosterhuis JA, Journée-de Korver HG, Kakebeeke-Kemme HM, et al. Transpupillary thermotherapy in choroidal melanomas. Arch Ophthalmol Transpupillary the

2 Oosterhuis JA, Journée-de Korver JG, Keunen JEE. Transpupillary thermotherapy. Results in 50 patients with choroidal melanoma. Arch Ophthalmol 1998;116:157-62.

3 Shields CL, Shields JA, Cater J, et al. Transpupillary thermotherapy for choroidal melanoma. Tumor control and visual results in 100 consecutive cases. Ophthalmology 1998;105:581-90.

4 Journée-de Korver JG, Oosterhuis JA, De Wolff-Rouendaal D, et al. Histopathological findings in human choroidal melanomas after transpupillary thermotherapy. Brf Ophthalmol 1997;81:234-9.
5 Collaborative Ocular Melanoma Study Group. Histopathologic characteristics of uveal melanomas in eyes enucleated from the colloborative ocular melanoma study COMS report no 6. Am f Ophthalmol 1998;125:745-66.
Kakebeeke-Kemme HM, Oosterhuis JA, de Wolff-Rouendaal D. Five-year Kakebeeke-Kemme HM, Oosterhuis JA, de Wolff-Rouendaal D. Five-year
follow-up study of choroidal and ciliary body melanomas after enucleation. follow-up study of choroidal and ciliary body melanomas after enucleation. 7 De Laey JJ, Hanssens M, Ryckaert S. Photocoagulation of malignant melanomas of the choroid. A reappraisal. Bull Soc Belge Ophtalmol 1986;213:9-18.

8 Diaz CE, Capone A, Grossniklaus HE. Clinicopathologic findings in recurogy 1998;105:1419-24.

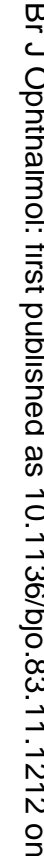

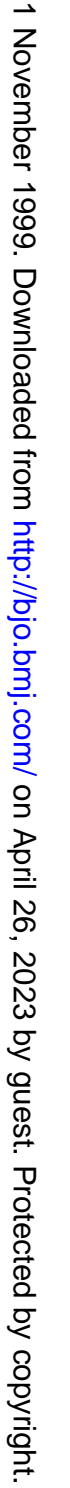

